\title{
Meta Distribution-optimal Base Station Deployment for Finite-Area Mobile Networks
}

\author{
Christodoulos Skouroumounis and Ioannis Krikidis \\ IRIDA Research Centre for Communication Technologies, \\ Department of Electrical and Computer Engineering, University of Cyprus, Cyprus \\ Email: \{cskour03, krikidis\}@ucy.ac.cy
}

\begin{abstract}
User mobility in cellular networks deployed in finite areas, results in the non-uniform spatial distribution of the mobile users (MUs), necessitating the effective deployment of base stations (BSs) in order to enhance the network connectivity. In this paper, we analyze the optimal non-uniform distribution of the BSs that provides ultra-reliable connectivity in the context of finite-area cellular networks, where the MUs employ the random waypoint (RWP) model. By using stochastic geometry tools, we first establish an analytical and tractable mathematical framework to investigate the uplink success probability in the context of non-uniform cellular networks. Furthermore, analytical expressions for the moments of the conditional success probability are derived, and a simple approximation of the meta-distribution (MD) is calculated, leveraging the moment-matching method with the Beta-distribution. Finally, we exploit the MD as a criterion for defining the optimal BS distribution, conditioned on the user's mobility. Our results reveal that the developed framework provides guidance in the design of cellular networks to efficiently determine the optimal network spatial deployment that offer ultra-reliable connectivity based on the mobility of MUs.
\end{abstract}

Index Terms-Random waypoint, meta-distribution, non uniform, stochastic geometry.

\section{INTRODUCTION}

Network densification via dense deployment of small cells, such as pico- and fempto-cells, is advocated as the key-enabling technology to enhance the network capacity and achieve high throughput [1]. Nevertheless, the existence of areas with heavy traffic load (also known as hotspots), which highly depends on the properties of the user's mobility, may compromise the network performance [2]. Hence, the modeling and the analysis of the user mobility in the context of small-cell networks is of paramount importance, as insightful design guidelines can be derived in order to ensure ultra-reliable connectivity.

Regarding the effect of the nodes mobility on the network performance, different mobility models are proposed. The most widely-used mobility model due to its tractability, is the random waypoint (RWP) model [3]. Authors in [4] evaluated the secrecy outage performance in the presence of moving interferers in a RWP model. For unmanned aerial vehicle (UAV) communications, the authors in [5] derived the statistics for the signal-to-interference ratio (SIR), in which multiple UAVs move according to the RWP model. Nevertheless, the abovementioned works assume infinite network area, and therefore, preserve the uniform properties of the node distribution. In

This work was supported by the Research Promotion Foundation, Cyprus, under the project INFRASTRUCTURES/1216/0017 (IRIDA). This work was also supported by the European Research Council (ERC) under the European Union's Horizon 2020 research and innovation programme (Grant agreement No. 819819). practice, the cellular networks, and especially the small-cells of HetNet deployments, have finite boundaries [6]. In such finite-area networks, whose nodes follow the RWP model, the resulting (steady-state) node distribution is far from uniform [7]. Because of that, the ability of a device to access the network and its observed interference are subjected to the device location. Many works showed that the employment of RWP-based mobility models result in the concentration of the nodes close to the center of the area. Thus, the knowledge of the actual node distribution is of critical importance to study the impact of the mobility on the performance metric of interest.

The above studies evaluate the considered performance metric i.e., coverage probability, throughput performance etc, at the typical link. While such metric is certainly important, it cannot reflect the performance variation among the individual users due to the spatial averaging involved. To overcome this limitation, the authors in [8] introduced the concept of metadistribution (MD), which provides a fine-grained information about the performance of the individual links. Therefore, such a metric can answer the critical question: "What fraction of devices in a finite-area cellular network achieve an SIR of $\theta$ with probability at least $x$, in a realization of the cellular network?", and hence to explore in depth how to exploit the node distribution in order to achieve ubiquitous connectivity.

Motivated by the above, in this paper, we evaluate the MD of the uplink (UL) SIR in the context of finite-area cellular networks with non-uniform base station (BS) deployment, where the users employ the RWP model. The main contribution of this paper, is the development of a novel mathematical framework that determines the optimal spatial distribution of the BSs in respect with the RWP-based movement of the mobile users (MUs), which leads to the maximum network connectivity. By leveraging tools from stochastic geometry, we derive the $b$-th moment of the conditional success probability of finitearea cellular networks, and a Beta-distribution approximation of the MD is calculated. Finally, we exploit the MD as a criterion for defining the optimal BS distribution, conditioned on the user's mobility. Numerical results unveil that there exists an optimal BS deployment that maximizes the network connectivity according to the MUs' mobility, highlighting the impact of non-uniform MU distributions.

\section{SySTEM MODEL}

\section{A. Network Model}

We consider the UL of a single-tier cellular network, where the network's nodes are confined in a circular disk $\mathcal{A} \in \mathbb{R}^{2}$ 


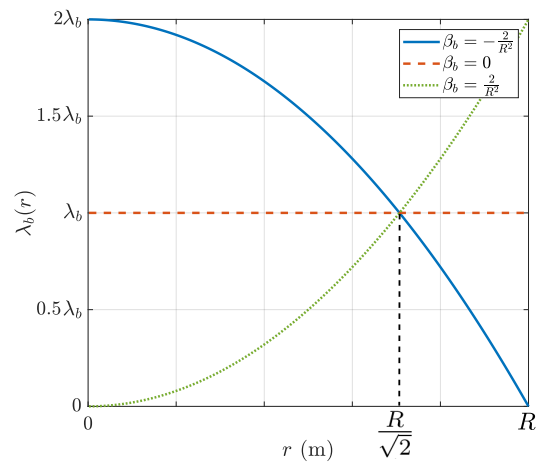

Fig. 1. Density function $\lambda_{b}(r)$ versus the distance $r(\mathrm{~m})$ for different distribution parameters $\beta_{b}=\left\{-\frac{2}{R^{2}}, 0, \frac{2}{R^{2}}\right\}$.

of area $|\mathcal{A}|=\pi R^{2}$ [2]. We model the locations of the BSs as a non-uniform Poisson point process (PPP) $\Phi_{b}$, of intensity $\lambda_{b}(r, \phi)$ in the circular disk $\mathcal{A}$ and zero intensity elsewhere. Although real-world network deployments are usually nonuniform, PPP-based spatial deployment is chosen to facilitate the analysis and can be regarded as a benchmark for evaluating the performance achieved with more sophisticated point processes. For simplicity, we focus on the analysis of quadratic radially symmetric BS distributions such that the intensity function is given by $\lambda_{b}(r)=\lambda_{b}\left(\alpha_{b}+\beta_{b} r^{2}\right), \quad 0 \leq r \leq R$, where $\lambda_{b}>0, \alpha_{b}=1-\beta_{b} R^{2} / 2$ and $\beta_{b} \in\left[-2 / R^{2}, 2 / R^{2}\right]$, such that there are, on average, $2 \pi \int_{0}^{R} \lambda_{b}(r) r d r=\lambda_{b}|\mathcal{A}|$ BSs in $\mathcal{A}$ [9]. As illustrated in Fig. 1, the parameter $\beta_{b}$ defines the BSs' deployment within $\mathcal{A}$, and allows us to interpolate between three different BSs deployment distributions, namely uniform $\left(\beta_{b}=0\right)$, near the border $\left(\beta_{b}>0\right)$, and near the center of the deployment region $\left(\beta_{b}<0\right)$. Based on Slivnyak's theorem [12], the BS at the origin becomes the typical BS under expectation over $\Phi_{b}$. Orthogonal access like orthogonal frequency-division multiple access is assumed i.e., each BS schedules only one MU on each resource block. MUs are spatially distributed according to a homogeneous PPP $\Phi_{u}$ of intensity $\lambda_{u}$. We consider the nearest-MU association rule i.e., the typical BS at the origin communicates with its closest MU.

All wireless signals are assumed to experience both largescale path-loss effects and small-scale fading. Specifically, the small-scale fading between two nodes is modeled by Rayleigh fading with unit average power, where different links are assumed to be independent and identically distributed. Hence, the power of the channel fading is an exponential random variable with unit mean, i.e. $h \sim \exp (1)$. For the large-scale path-loss, we assume an unbounded singular path-loss model, $L(X, Y)=\|X-Y\|^{-a}$ which assumes that the received power decays with the distance between the transmitter at $X$ and the receiver at $Y$, where $a>2$ is the path-loss exponent.

\section{B. User Mobility Model}

The process representing the movement of a MU within the finite area $\mathcal{A} \in \mathbb{R}^{2}$ can be described as follows. Initially, each MU is placed at the point $P_{1}$ chosen from the uniform distribution $\Phi_{u} \in \mathcal{A}$. Then, each MU uniformly chooses a destination (also called waypoint) $P_{2}$ in the region $\mathcal{A}$ and moves towards it with randomly selected speed i.e., $u \in$ $\left[u_{\min }, u_{\max }\right]$, which remains constant during that movement. A new direction and speed are chosen only after the MU reaches the destination. It is important to mention here that, the MUs bounce back when they reach the boundary, and hence the number of MUs in $\mathcal{A}$ remains constant i.e., $\lambda_{u}|\mathcal{A}|$. Therefore, a MU starting near the boundaries of the network area clearly finds more destination waypoints in directions toward the center of the area than toward the border. As time passes and the MUs perform a number of movement periods, the spatial distribution of the MUs becomes more and more non-uniform. Finally, for a long running time of the movement process, a stationary distribution, also known as steady-state distribution, is achieved [3]. The intensity measure of the above-mentioned steady-state distribution of MUs follows as $\Lambda_{u}(B(o, r))=\lambda_{u} \pi r^{2}\left(\alpha_{u}+\beta_{u} \frac{r^{2}}{2}\right)$, where $r \leq R$, and the intensity function is, thus, given by

$$
\lambda_{u}(r) \triangleq \lambda_{u}^{\infty}(r)=\lambda_{u}\left(\alpha_{u}+\beta_{u} r^{2}\right), \quad 0 \leq r \leq R,
$$

where $\lambda_{u}^{\infty}(r)$ is the intensity function of the steady-state of the MUs' distribution, $\alpha_{u}=1-\beta_{u} R^{2} / 2$ and $\beta_{u} \in\left[-2 / R^{2}, 2 / R^{2}\right]$. Note that, the special case $\beta_{u}=2 / R^{2}$ refers to the RWP on border (RWPB) ${ }^{1}$ mobility model, while the case where the MUs are static and therefore uniformly distributed i.e., $u=0$, is capture by $\beta_{u}=0$. Then, the probability density function (pdf) of the distance $R$ between the typical BS and its serving $\mathrm{MU}$, is given by [3]

$$
f_{R}(r)=2 \pi \lambda_{u} r\left(\alpha_{u}+\beta_{u} r^{2}\right) \exp \left(-\pi \lambda_{u} r^{2}\left(\alpha_{u}+\beta_{u} \frac{r^{2}}{2}\right)\right) .
$$

\section{Meta-distribution of SIR}

The MD has been introduced as a performance metric that provides a more complete spatial distribution rather than merely spatial averages as performed in the majority of works in the literature. The MD of the SIR is the distribution of the conditional success probability given a realization of the PPP. Specifically, the MD of the SIR is a two-parameter distribution function, defined as

$$
\bar{F}_{P_{s}(\theta)}(\tau)=\mathbb{P}_{o}^{!}\left[P_{s}(\theta)>\tau\right], \quad \theta \in \mathbb{R}^{+}, \tau \in[0,1],
$$

where $P_{s}(\theta)$ s the success probability conditioned on a PPP $\Phi$, i.e. $P_{s}(\theta)=P[\mathrm{SIR}>\theta \mid \Phi]$ and $\mathbb{P}_{o}^{!}$is the reduced Palm probability [8]. For our network setup, the UL SIR at the typical $\mathrm{BS}$, is given by

$$
\mathrm{SIR} \triangleq \frac{h_{x_{0}}\left\|x_{0}\right\|^{-a}}{\sum_{x \in \Phi_{I}} h_{x}\|x\|^{-a}},
$$

where $x_{0}$ is the location of the MU which communicates with the typical BS, $h_{x}$ is the power of the channel fading between the typical BS and the MU at $x$, and $\Phi_{I} \subset \Phi_{u} \backslash\left\{x_{0}\right\}$ represents the point process of active interfering MUs (see Section III-A).

\section{Analytical Results for the Meta-Distribution}

In this section, we first characterize the received interference at the typical BS, and then derive the moments of the conditional success probability. We provide analytical expressions

\footnotetext{
${ }^{1}$ RWPB model is a special case of RWP, where the destination points are chosen uniformly on the border of $\mathcal{A}$.
} 
which will be useful for computing the MD of the UL SIR in Section IV. Throughout this paper, we will denote by $r_{x}$ the distance between the origin and a MU that is located at $x \in \Phi_{u}$, i.e. $r_{x}=\|x\|$.

\section{A. Interference Characterization}

Firstly, we investigate the statistical properties of $\Phi_{I}$ which is the non-uniform distribution of the active interfering MUs. The locations of the active MUs can be seen as a Voronoi perturbed lattice process [10]. However, the analysis of the network performance with such approach is not mathematically tractable. Therefore, for tractability purposes, the aggregate interference seen at the typical BS is approximated by the interference seen from a non-homogeneous PPP [11], which is characterized in the following lemma.

Lemma 1. The active interfering MUs follow a non homogeneous PPP $\Phi_{I}$ with density function $\lambda_{u}^{I}(r)$, that is given by

$$
\lambda_{u}^{I}(r)=\lambda_{u}(r) \delta(r)\left(1-\exp \left(-\pi \lambda_{u}(r) \delta(r) r^{2}\right)\right),
$$

where $\delta(r)$ represents the load factor of a cell at distance $r$, which is equal to

$$
\delta(r)=\frac{\gamma[K, \pi \Omega(r)]}{\Gamma[K]}-\frac{\Omega(r)^{K}}{\Gamma[K]} \frac{\gamma\left[K, \pi\left(\lambda_{u}(r) R^{2}+\Omega(r)\right)\right]}{\left(R^{2} \lambda_{u}(r)+\Omega(r)\right)^{K}},
$$

$\Omega(r)=K R^{2} \lambda_{b}(r), K=3.575$, and $\gamma[\cdot, \cdot]$ denotes the lower incomplete gamma function.

Proof. See Appendix A.

Recall that $\lambda_{f}(r)=\lambda_{f}\left(1-\beta_{f} R^{2} / 2+\beta_{f} r^{2}\right)$ is the density function of the nodes that belong in the non-homogeneous PPP $\Phi_{f}$, where $f=\{b, u\}$. We simplify the analysis by considering the asymptotic case where $\lambda_{u} \rightarrow \infty$, which is in line with the expected huge proliferation of end-user devices in future communication systems. Hence, by approximating the above densities by a step function i.e.,

$$
\tilde{\lambda}_{f}(r)= \begin{cases}2 \lambda_{f} \mathbb{1}_{r<\mathcal{R}} & \text { if } \beta_{f}<0 \\ 2 \lambda_{f} \mathbb{1}_{r>\mathcal{R}} & \text { if } \beta_{f}>0\end{cases}
$$

where $\mathcal{R}$ represents the maximum (minimum) distance of a node $x \in \Phi_{f}$ from the origin for the case $\beta_{f}<0\left(\beta_{f}>0\right)$, where $f \in\{b, u\}$, and $\mathbb{1}_{X}$ denotes the indicator function, where $\mathbb{1}_{X}=1$ if $X$ holds; otherwise $\mathbb{1}_{X}=0$. Based on the Fig. 1, we assume $\mathcal{R}=R / \sqrt{2}$ and $\beta_{u}=\beta_{b}=\beta$. The validity of the assumptions will be shown in the numerical results.

Proposition 1. In an ultra-dense MU deployment, the intensity of the active interfering MUs, can be re-written as

$$
\widetilde{\lambda}_{u}^{I}(r)=\frac{\left(4 K R^{2} \lambda_{u} \lambda_{b}\right)^{K}}{(1+K) \Gamma[K]} \exp \left(-2 \pi K R^{2} \lambda_{b}\right) \Delta(r),
$$

where $\Delta(r)=\mathbb{1}_{r<\mathcal{R}}$ if $\beta<0$, otherwise $\Delta(r)=\mathbb{1}_{r>\mathcal{R}}$.

Proof. Based the inequality $\kappa x^{-\kappa} \gamma(\kappa, x) \leq \frac{1+\kappa \exp (-x)}{1+\kappa}$, the upper-bound for the load factor can be derived.

\section{B. Moments of Conditional Success Probability}

From the definition, the $b$-th moment of the MD is given by $M_{b}(\theta) \triangleq \mathbb{E}\left(P_{s}(\theta)^{b}\right)$. The following Lemma provides the analytical expressions for the moments of the conditional success probability.
Lemma 2. The b-th moment $M_{b}$ of the conditional success probability for the typical BS in UL finite-area cellular networks with user mobility, is given by

$$
M_{b}=\int_{0}^{R} \exp \left(\int_{0}^{R} \frac{1-\left(1+\theta \frac{r^{a}}{u^{a}}\right)^{b}}{\left(1+\theta \frac{r^{a}}{u^{a}}\right)^{b}} 2 \pi v \lambda_{u}^{I}(v) \mathrm{d} v\right) f_{R}(r) \mathrm{d} r,
$$

where $f_{R}(r)$ denote the pdf of the distance between a BS and its serving $M U$, which is given by the expression (2).

Proof. By using the moment generating function of an exponential random variable [12], the conditional UL success probability. is given by $P_{s}(\theta)=\prod_{x \in \Phi_{I}} 1 /\left(1+\theta\left(\frac{r_{x}}{r_{x_{0}}}\right)^{-a}\right)$. Then, the $b$-th moment of the conditional success probability, $M_{b}$, can be written as

$$
M_{b} \approx \mathbb{E}_{r_{x_{0}}} \exp \left(\int_{0}^{R} \frac{1-\left(1+\theta \frac{r_{x_{0}}^{a}}{r_{x}^{a}}\right)^{b}}{\left(1+\theta \frac{r_{x_{0}}^{a}}{r_{x}^{a}}\right)^{b}} 2 \pi v \lambda_{u}^{I}(v) \mathrm{d} v\right),
$$

where (9) follows from (5) and the probability generating functional of PPP. By un-conditioning on $r_{x_{0}}$ the derived expression using (2), we conclude to the desired expression.

Even through the expressions in Lemma 2 can be evaluated using numerical tools, this could be difficult due to the presence of multiple integrals. To address this, we simplify the analysis by employing the assumptions used for the Proposition 1 and also consider the special case where $a=4$.

Lemma 3. In an ultra-dense $M U$ deployment and $a=4$, the $b$-th moment $M_{b}$ of the conditional success probability for the case $\beta<0$, can be re-written as

$$
\begin{aligned}
\widetilde{M}_{b}=2 \pi \sqrt{b \theta} & \frac{\left(4 K R^{2} \lambda_{u} \lambda_{b}\right)^{K}}{(1+K) \Gamma[K]} \exp \left(-2 \pi K R^{2} \lambda_{b}\right) \\
& \times \int_{0}^{\mathcal{R}} r^{2} \arctan \left[\frac{\mathcal{R}^{2}}{r^{2} \sqrt{b \theta}}\right] \widetilde{f}_{R}(r) \mathrm{d} r
\end{aligned}
$$

and for the case $\beta>0$, is given by

$$
\begin{aligned}
& \widetilde{M}_{b}=2 \pi \sqrt{b \theta} \frac{\left(4 K R^{2} \lambda_{u} \lambda_{b}\right)^{K}}{(1+K) \Gamma[K]} \exp \left(-2 \pi K R^{2} \lambda_{b}\right) \\
& \times \int_{\mathcal{R}}^{R} r^{2}\left(\arctan \left[\frac{R^{2}}{r^{2} \sqrt{b \theta}}\right]-\arctan \left[\frac{\mathcal{R}^{2}}{r^{2} \sqrt{b \theta}}\right]\right) \tilde{f}_{R}(r) \mathrm{d} r,
\end{aligned}
$$

where

$$
\tilde{f}_{R}(r)= \begin{cases}4 \pi \lambda_{u} r \exp \left(-2 \pi \lambda_{u} r^{2}\right) & \text { if } \beta<0, \\ 4 \pi \lambda_{u} r \exp \left(-2 \pi \lambda_{u}\left(r^{2}-\mathcal{R}^{2}\right)\right) & \text { if } \beta>0,\end{cases}
$$

and $\arctan [\phi]$ is the inverse of the tangent function.

Proof. Based on the approximated intensity function $\widetilde{\lambda}_{u}(r)$, the pdf can be calculated as $\widetilde{f}_{R}(r)=\mathrm{d}[1-\exp (-\Lambda(r))] / \mathrm{d} r$, where $\Lambda(r)$ is the intensity function, and is given by $\Lambda(r)=$ $2 \pi \lambda_{u} \int_{0}^{r} v \Delta(v) \mathrm{d} v$, where $\Delta(\cdot)$ is given in Proposition 1. Then, based on the Bernoulli inequality i.e., $(1+x)^{y} \geq 1+y x$ where $x>-1$, the moments of the conditional success probability can be simplified. Due to space limitations, the simplified expression is omitted. Finally, the desired expressions in Lemma 3 can be derived by substituting $a=4$ and by un-conditioning on $r_{x_{0}}$ with the aid of $\widetilde{f}_{R}(r)$. 


\section{Meta-Distribution And Optimal Deployment}

In this section, we evaluate the MD of the SIR to characterize the connectivity reliability when MUs employ the RWP model in finite-area networks. This fine-grained performance metric provides the information about what fraction of MUs in each realization of the point process can successfully decode the received signal with probability at least $\tau$. Finally, the optimal deployment of the small cells that maximizes the MD of the SIR is investigated, aiming to provide ultra-reliable connectivity for the MUs.

\section{A. Meta Distribution and its Beta Approximation}

According to the Gil-Pelaez theorem [8], the MD can be calculated as

$$
\bar{F}_{P_{s}(\theta)}(\tau)=\frac{1}{2}+\frac{1}{\pi} \int_{0}^{\infty} \frac{\operatorname{Im}\left[\exp \left(-j t \log x M_{j t}\right)\right]}{t} \mathrm{~d} t,
$$

where $\operatorname{Im}[z]$ denotes the imaginary parts of $z \in \mathbb{C}$. Authors in [8] showed that the MD can be approximated by matching the mean and variance of the Beta-distribution with $M_{1}$ and $M_{2}$ given in Lemma 2. The first and second moments of a betadistributed random variable $X$ with shape parameters $\eta, \epsilon>0$, are given by $\mathbb{E}[X]=\eta /(\eta+\epsilon)$ and $\mathbb{E}\left[X^{2}\right]=(\eta+1) /(\eta+\epsilon+$ $1)$. The following theorem provides the MD of our considered network setup.

Theorem 1. The MD is approximated by the beta distribution for the considered network deployments, as

$$
\bar{F}_{P_{s}(\theta)}(x) \approx 1-B(\eta, \epsilon)^{-1} \int_{0}^{x} t^{\eta-1}(1-t)^{\epsilon-1} d t .
$$

where $B(\cdot, \cdot)$ is the Beta function, and

$$
\eta=\frac{M_{1} M_{2}-M_{1}^{2}}{M_{1}^{2}-M_{2}}, \quad \epsilon=\frac{\left(1-M_{1}\right)\left(M_{2}-M_{1}\right)}{M_{1}^{2}-M_{2}} .
$$

where $M_{1}$ and $M_{2}$ are given in Lemma 2 .

\section{B. Meta-Distribution Optimal Deployment}

From the network operator point-of-view, the deployment of the small cells focuses on eliminating any coverage hole in outdoor and indoor environments, so their distribution and density must ensure ubiquitous connectivity. Since the increase of the number of small cells is a prohibitively expensive approach, we intend to maximize the network connectivity by effectively deploying the small cells based on the traffic density (i.e. hotspots) of the network. Hence, in this section, we investigate the optimal distribution parameter of the BSs that provides ultra-reliable connectivity for the considered network setup. Specifically, based on the RWP-based movement of the MUs, we examine the parameter of the non-uniform distribution of the BSs that maximizes the achieved MD of the UL SIR.

Let $\beta_{b}^{*}$ represents the distribution parameter of the BSs that maximizes the expression (13) i.e.,

$$
\beta_{b}^{*}\left(\beta_{u}, \lambda_{b}, \lambda_{u}\right)=\underset{\beta_{b}}{\arg \max } \bar{F}_{P_{s}(\theta)}(x),
$$

conditioned on $\beta_{u}$, and the densities of the MUs $\lambda_{u}$ and the BSs $\lambda_{b}$. Particularly, the equation (14) is a maximization over multiple integrals, and is therefore computationally difficult. As a result, an exact closed-form solution cannot be obtained and the problem should therefore be tackled numerically.

\section{NUMERICAL RESULTS}

The spatial densities of the BSs and the MUs are $\lambda_{b}=10^{-4}$ and $\lambda_{u}=10^{-4}$, respectively, the path-loss exponent is set to $a=4$ and $R=500 \mathrm{~m}$.

Fig. 2 illustrates the effect of the MU's mobility on the MD of the UL SIR for different reliability values $\tau$, where the BSs are uniformly distributed in the area $\mathcal{A}$, i.e. $\beta_{b}=0$. Specifically, we plot the approximated MD, that is given by (13), versus the decoding threshold $\theta(\mathrm{dB})$ for different reliability values $\tau=\{0.1,0.9\}$ and mobility models $\beta_{u}=$ $\left\{0,-2 / R^{2}, 2 / R^{2}\right\}$. Firstly, Fig. 2 shows the good agreement between the theoretical (solid, dashed and dotted lines) and the simulation (markers) results, validating our analysis and verifying the accuracy of the approximation of the moments given in Lemma 2. An important observation from this figure is that, for each reliability value $\tau$, the mobility of the MUs causes the reduction of the achieved MD of the uniformly deployed BSs, compared with the case where the MUs are static i.e., $\beta_{u}=0$. This was expected since, the RWP-based mobility of the MUs in finite-area networks, results in the MUs' concentration near the center $\left(\beta_{u}=-2 / R^{2}\right)$ or the borders $\left(\beta_{u}=2 / R^{2}\right)$ of the network. As a result, the number of BSs whose Voronoi-cell is empty of MUs i.e., inactive BSs, is increased, and hence, the percentage of uniformly distributed BSs that successfully decode the received signal is reduced. This observation highlights the demand of a nonuniform deployment of BSs in order to address the negative effect of the MUs mobility on the network performance.

Fig. 3 reveals the impact of MUs' density on MD of the UL SIR for different spatial distribution parameters $\beta_{u}$, where $\beta_{u}=\left\{0,-2 / R^{2}, 2 / R^{2}\right\}$. Specifically, Fig. 3 demonstrates the network performance that can be achieved both by our proposed scheme (i.e. $\beta_{b}=\beta_{b}^{*}$ ) and a conventional scheme (i.e. $\beta_{b}=\beta_{u}$ ). It is interesting to note that at low MUs' densities, the increasing number of MUs improves the network performance. However, by increasing the MUs' density beyond a critical point, the network performance decreases. This observation is based on the fact that at low density values, the number of BSs with at least one MU in their serving area becomes larger and therefore, the fraction of BSs that successfully achieve an SIR of $\theta(\mathrm{dB})$ increases. In contrast, for high MUs' densities, the overall interference caused by the active MUs increases, thereby reducing the ability of the BSs to decode the received signal and consequently, the fraction of BSs that successfully achieve an SIR of $\theta(\mathrm{dB})$ decreases. Moreover, for the ultradense MU deployments, the network performance converges to a constant floor. This observation was expected, since both the number of active interfering MUs and the overall network interference remain constant, due to the assumption that each BS schedules only one MU on each resource block. Fig. 3 shows that, the adoption of the simplified model for nodes' density functions provides lower complexity methodology for evaluating the system performance in the context of ultra-dense deployments, without being significantly deficient in accuracy. Finally, Fig. 3 reveals that the performance achieved by our 


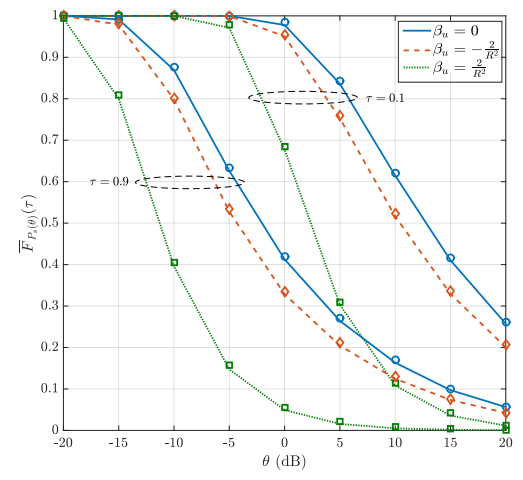

Fig. 2. MD versus $\theta(\mathrm{dB})$ for different reliability values $\tau=\{0.1,0.9\}$ and MUs' mobility models $\beta_{u}=\left\{0,-2 / R^{2}, 2 / R^{2}\right\} ; \beta_{b}=0$.

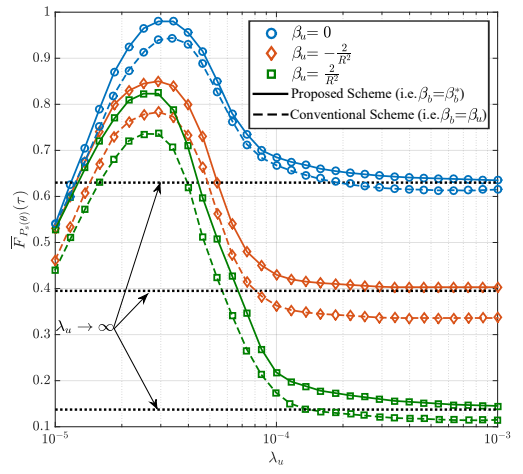

Fig. 3. MD versus the MUs' density $\lambda_{u}$ for different spatial distribution parameters $\beta=$ $\left\{0,-2 / R^{2}, 2 / R^{2}\right\} ; \beta=\beta_{b}=\beta_{u}, \theta=0 \mathrm{~dB}$, $\tau=0.5$.

proposed scheme outperforms that of the conventional scheme, highlighting the importance of our proposed technique for achieving ultra-reliable connectivity.

Fig. 4 shows the optimal deployment of BSs $\beta_{b}^{*}$ that maximizes the MD as a function of the non-uniform MU distribution parameter $\beta_{u}$, for different ratios of densities i.e., $\lambda_{u} / \lambda_{b}$. We can easily observe that, for RWP-based mobility models with $\beta_{u}>0$, the optimal $\mathrm{BS}$ distribution parameter is also $\beta_{b}^{*}>0$. On the other hand i.e., $\beta_{u}<0$, the optimal BS distribution parameter depends on $\beta_{u}$ and the ratio $\lambda_{u} / \lambda_{b}$. As we can easily observe, by increasing the ratio $\lambda_{u} / \lambda_{b}$, the optimal BS distribution parameter $\beta_{b}^{*}$ decreases for a given MU distribution parameter $\beta_{u}$.

\section{CONCLUSION}

In this paper, we studied the MD of the UL SIR for finitearea non-uniform cellular networks, where the MUs employ the RWP model. Specifically, based on the MUs' mobility, we derived the optimal parameter of the spatial distribution of BSs, which maximizes the achieved MD. By applying tools from stochastic geometry theory, the moments of the conditional success probability were derived analytically and the actual MD was approximated using the moment-matching method for the Beta-distribution. Our results highlight the impact of the MUs mobility on the optimal BS distribution, providing guidance for the planning of cellular networks in order to achieve ultra-reliable connectivity. A future extension of this work is the consideration of multi-cell heterogeneous deployments and investigate spatially correlated finite area cellular networks.

\section{APPENDIX A \\ PROOF OF LEMMA 1}

The probability density function (pdf) of the size of a Voronoi cell at distance $r$ from the origin, is accurately predicted by a Gamma distribution [10]

$$
f_{A}(x, r)=\left(\lambda_{b}(r)\right)^{K} \frac{K^{K}}{\Gamma[K]} x^{K-1} \exp \left(-K \lambda_{b}(r) x\right),
$$

where where $A$ is a random variable that denotes the size of the Voronoi cell, $K=3.575$ and $\Gamma[\cdot]$ denotes the Gamma function. For tractability purposes, we consider a constant MUs' intensity for a small area around a random point. Then,

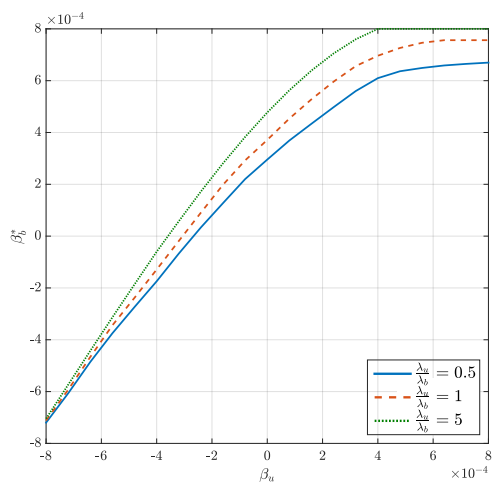

Fig. 4. Optimal BS distribution parameter $\beta_{b}^{*}$ versus the MU distribution parameter $\beta_{u}$ for different ratio of $\lambda_{u} / \lambda_{b} ; \lambda_{b}=10^{-4}, \theta=0 \mathrm{~dB}$, and $\tau=0.5$.

the load factor $\delta(r)$ is obtained by first writting the probability of having $n$ number of MUs in a cell with cell-size $A$ given by $P_{A}(n)=\frac{\left(\lambda_{u}(r) A\right)^{n}}{n !} \exp \left(-\lambda_{u}(r) A\right)$, solving it for $n \neq 0$, and then integrating it over the distribution of A. Hence, the load factor $\delta(r)$, is given by

$$
\delta(r)=\int_{0}^{\pi R^{2}} \frac{\left(K x \lambda_{b}(r)\right)^{K}\left(1-\exp \left(-x \lambda_{u}(r)\right)\right)}{\exp \left(K x \lambda_{b}(r)\right) x \Gamma[K]} \mathrm{d} x,
$$

In order to account the fact that a single MU is associated with each BS, the resulting intensity $\lambda_{u}(r) \delta(r)$ must be appropriately thinned with the probability $\left(1-\exp \left(-\pi \lambda_{u}(r) \delta(r) r^{2}\right)\right)$ [10]. Hence the expressions of Lemma 1 are derived.

\section{REFERENCES}

[1] A. Damnjanovic et al., "A survey on 3GPP heterogeneous networks," IEEE Wireless Commun., vol. 18, no. 3, pp. 10-21, June 2011.

[2] C. Bettstetter, G. Resta, and P. Santi, "The node distribution of the random waypoint mobility model for wireless ad hoc networks," IEEE Trans. Mobile Computing, vol. 2, no. 3, pp. 257-269, Sept. 2003.

[3] Z. Gong and M. Haenggi, "Interference and outage in mobile random networks: Expectation, distribution, and correlation," IEEE Trans. Mobile Computing, vol. 13, no. 2, pp. 337-349, Feb. 2014.

[4] N. Cao, Y. Chen, and Z. Yang, "Secrecy outage probability with randomly moving interferers in Nakagami-m fading," IEEE Commun. Lett., vol. 23, no. 1, pp. 76-79, Jan. 2019.

[5] P. K. Sharma and D. I. Kim, "Coverage probability of 3D mobile UAV networks," IEEE Wireless Commun. Lett., vol. 8, no. 1, pp. 97-100, Feb. 2019.

[6] K. Koufos and C. P. Dettmann, "Temporal correlation of interference and outage in mobile networks over one-dimensional finite regions," IEEE Trans. Mobile Computing, vol. 17, no. 2, pp. 475-487, Feb. 2018.

[7] E. Hyytia, P. Lassila, and J. Virtamo, "Spatial node distribution of the random waypoint mobility model with applications," IEEE Trans. Mobile Computing, vol. 5, no. 6, pp. 680-694, June 2006.

[8] M. Haenggi, "The meta distribution of the SIR in poisson bipolar and cellular networks," IEEE Trans. Wireless Commun., vol. 15, no. 4, pp. 2577-2589, Apr. 2016.

[9] P. Pratt, C. P. Dettmann, and O. Georgiou, "Optimal non-uniform deployments in ultra-dense finite-area cellular networks," IEEE Commun. Let., vol. 21, no. 5, pp. 1139-1142, May 2017.

[10] S. Singh, X. Zhang, and J. G. Andrews, "Joint rate and SINR coverage analysis for decoupled uplink-downlink biased cell associations in HetNets," IEEE Trans. Wireless Commun., vol. 14, no. 10, pp. 5360-5373, Oct. 2015.

[11] C. Skouroumounis, C. Psomas, and I. Krikidis, "Heterogeneous FDmm-Wave cellular networks with cell center/edge users," IEEE Trans. Commun., vol. 67, no. 1, pp. 791-806, Jan. 2019.

[12] M. Haenggi, Stochastic geometry for wireless networks, in Cambridge, U.K.: Cambridge Univ. Press, 2012. 